\title{
Sources of "overadditivity" in prism adaptation
}

\author{
GORDON M. REDDING \\ Illinois State University, Normal, Illinois 61761 \\ and \\ BENJAMIN WALLACE \\ Western Illinois University, Macomb, Illinois 61455
}

\begin{abstract}
In two experiments, tests of visual shift (VS), proprioceptive shift (PS), and negative aftereffect (NA) were made following 15-min exposure to 20-D (base-right) displacement. In Experiment 1, subjects engaged in saggital pointing (hand exposure) at single or multiple (three) exposure targets, while in Experiment 2, subjects experienced hand exposure or hallway exploration (hall exposure) factorially combined with single or multiple (three) NA test targets. In both experiments, additivity (VS+PS = NA) appeared for multiple target conditions while single target conditions produced "overadditivity" (VS+PS $>$ NA). This additivity effect for target conditions did not interact with exposure conditions in Experiment 2, although VS was greater in hall exposure and PS was greater in hand exposure. The presence of additivity with multiple targets was supported by a higher correlation between the NA test and each component test with multiple targets in both experiments. These results are interpreted as indicating two causes of overadditivity: specific motor response learning with single-exposure targets and association of a single NA test target with a cognitive shift in egocentric straight-ahead.
\end{abstract}

Considerable evidence presently supports the idea that perceptual change occurs in multiple loci during exposure to an optically displaced visual field, and that these adaptive components combine in a simple linear or additive manner to produce the total adaptation occurring in the sensory-motor control loop regulating our interaction with the world (e.g., Hay \& Pick, 1966; McLaughlin \& Webster, 1967; Wallace, 1977; Wallace \& Garrett, 1975; Wilkinson, 1971). Exceptions to this general conclusion have usually taken the form of what may be called "underadditivity." That is, the sum of component adaptation fails to reach the level of adaptation measured for the complete control loop. For example, the sum of tests measuring visual shift (VS) and proprioceptive shift (PS) is less than adaptation measured by a common negative-aftereffect (NA) test, which is presumed to be sensitive to both visual and proprioceptive change (i.e., VS + PS $<$ NA). Such underadditivity has usually been explained by postulating additional adaptive components (e.g., Templeton, Howard, \& Wilkinson, 1974; Welch, Choe, \& Heinrich, 1974) and has not seriously challenged the "additivity" hypothesis.

A preliminary report of the data in this study was presented at the meeting of the Psychonomic Society, Washington, D. C., November 1972. The authors gratefully acknowledge the assistance of Tracy Knight in data collection. The second author is now at Cleveland State University, Cleveland, Ohio. Requests for reprints should be addressed to Gordon M. Redding, Department of Psychology, Illinois State University, Normal, Illinois 61761 .
In a recent parametric study of component adaptation, Redding and Wallace (1976) found evidence of another kind of additivity failure which might be called "overadditivity." For a variety of exposure conditions and during both acquisition and decay, the sum of visual and proprioceptive tests tended to exceed the common test (i.e., VS + PS $>$ NA). For example, such overadditivity was especially obvious and significant with hall exposure, where subjects explored hallways while wearing displacing prisms. However, this effect was also numerically present with hand exposure, which involved the more usual eye-hand coordination task during prism exposure, and the effect was not modified whether lateral or saggital pointing was employed. The reliability of this effect across variations in exposure and test conditions argues against its being a spurious result, and the present study more firmly established the reality of such overadditivity and identified its causal bases.

Experiment 1 investigated hand exposure and tested the hypothesis that the single-exposure target employed by Redding and Wallace (1976) promoted acquisition of a specific motor response (e.g., Harris, 1963) that was evoked when subjects were tested by pointing straight ahead in the absence of any visual target (i.e., the PS test). If this acquired motor response was in the adaptive direction, it should lead to an inflated measure of PS and should appear as overadditivity. Thus, in the first experiment, the number of exposure targets was manipulated to test this hypothesis.

Experiment 2 investigated both hand and hall expos- 
ure, and tested another explanation or overadditivity originally suggested by Redding and Wallace. This hypothesis is based on the "straight-ahead" shift outlined by Harris (1974). For example, the apparent asymmetry of the subject in the environment when wearing displacing prisms may produce a cognitive shift in straight-ahead in the direction of displacement, in addition to any true perceptual recalibration of egocentric direction. As originally formulated by Harris, such a straight-ahead shift would not produce overadditivity, because the NA test, which requires pointing at a target without reference to straight-ahead, would not be affected. Also, the effect of the straight-ahead shift on the VS and PS tests should be of equal, but opposite, magnitudes (i.e., spurious "adaptation" on the VS test and "maladaptive" change on the PS test). However, with a single test target as used by Redding and Wallace, the subject may form an association between the straight-ahead position in space and the position of the NA test target, assuming them to be the same. Thus, if the subject tends to point "straight ahead" rather than at the target per se in the NA test, magnitude of the NA would be reduced, leading to the overadditivity result. The second experiment manipulated the number of NA test targets to test for the formation of such an association between target position and cognitive straight-ahead.

\section{EXPERIMENT 1}

In addition to manipulating number of exposure targets, Experiment 1 also varied whether the exposure and test field was uniformly illuminated, i.e., a clearly delimited field, or uniformly dark with effectively no limits. In their first experiment, with an illuminated test field, Redding and Wallace (1976) found very little VS, while with a dark test field in the second experiment VS was substantial. They speculated that this effect might be due to a tendency for subjects to center the visual target within the available test field, thereby reducing the measured magnitude of VS when the test field is illuminated. The dark vs. light environment manipulation in the present experiment was intended as a more direct test of this speculation. To minimize any distracting differences between exposure and test, the same illumination condition was maintained for both exposure and test fields.

\footnotetext{
Method

Subjects. Subjects were 40 male and female volunteers from Western Illinois University, who received extra course credit for participation. Apparatus constraints required only righthanded individuals, and only those with normal or correctedto-normal vision (subject report) were used.

Procedure. During a 15-min exposure, subjects marked targets with the visible hand. The apparatus was the same as that used by Redding and Wallace (1976, Experiment 1), except saggital instead of lateral movement was used to mark targets. Separate groups pointed at a single optically straight-ahead
}

target, or at three simultaneously visible targets optically straight ahead and $5^{\circ}$ left and right of straight-ahead. Targets were white vertical strips $\left(4^{\circ} \times 15.5^{\circ}\right)$, and position was adjusted to compensate for the monocular, base-right, 20-D displacement, such that targets appeared optically and phenomenally symmetrical in the visual field, but of course physically disparate. Subjects made a single pointing movement, with no lateral error correction, but pointing was accurate by the end of exposure. Pointing was paced by metronome (1-sec rate) and order of pointing at multiple targets was randomized for each subject such that pointing was equally frequent at each target.

Single and multiple exposure targets were also factorially combined with two kinds of exposure/test environments, producing four groups of 10 subjects each. In the light environment, the visual field was normally illuminated, and in the dark environment illumination was by "black" light. In the dark condition, the low-reflectance (flat-black) background was effectively nonvisible, while in the light condition, the background as well as hand and target(s) were visible. In both environments the hand was visible through most of the movement.

Level of adaptation was defined as the difference between pretests and posttests, and three kinds of tests were performed (five measures of each in a random order) without displacement. ${ }^{1}$ To test for VS, subjects verbally indicated when a laterally moved target (starting position randomly $15^{\circ}$ left or right of straight-ahead) appeared to be in the straight-ahead position. As a test for PS, subjects simply pointed straight ahead in the absence of any visual stimulus. The common test consisted of the usual NA eye-hand coordination test, pointing with the unseen hand at a single straight-ahead target.

\section{Results and Discussion}

Data for individual subjects and relevant means, together with confidence limits, are shown in Table 1. An analysis of variance indicated only a significant main effect for the kind of test, $F(2,72)=87.65$, $\mathrm{p}<.001$, and a significant interaction of tests and number of exposure targets, $F(2,72)=8.81, p<.001$. Subsequent comparisons revealed virtually perfect additivity (VS $+\mathrm{PS}=4.04^{\circ}, \mathrm{NA}=4.06^{\circ}$ ) with multiple targets, $t(19)=-.41$, but significant overadditivity $\left(\mathrm{VS}+\mathrm{PS}=4.93^{\circ}, \mathrm{NA}=3.82^{\circ}\right.$, difference $\left.=1.11^{\circ}\right)$ with a single-exposure target, $t(19)=4.19, \mathrm{p}<.001$. This difference between single and multiple targets is particularly striking, since it is reflected in the data for individual subjects. With single targets, 18 of the 20 subjects showed overadditivity, while with multiple targets only half the subjects showed overadditivity. Moreover, deviation from additivity for individuals in the multiple target conditions was quite small, indicating random variation around zero, while overadditivity was substantial for most subjects in single-target conditions.

The conclusion of greater additivity with multiple targets is further supported by rank-order correlational analysis. As would be expected from the additivity hypothesis, the NA test correlated more highly with VS (.70) and PS (.73) in multiple-target conditions than in single-target conditions (coefficients of .47 and .48 , respectively). (The coefficients were significantly greater than zero in both conditions, but the probability level was .001 with multiple targets and 
Table 1

Level of Adaptation in Degrees for Conditions of Singleand Multiple-Exposure Targets, Combined with Dark and Light Environment

\begin{tabular}{|c|c|c|c|c|c|c|c|c|}
\hline & \multicolumn{4}{|c|}{ Single Target } & \multicolumn{4}{|c|}{ Multiple Targets } \\
\hline & VS & PS & NA & D & VS & PS & NA & D \\
\hline & \multicolumn{8}{|c|}{ Dark Environment } \\
\hline & $\begin{array}{r}.6 \\
.5 \\
.8 \\
1.1 \\
1.9 \\
1.1 \\
2.1 \\
.8 \\
2.3 \\
2.9\end{array}$ & $\begin{array}{l}3.3 \\
3.6 \\
4.5 \\
4.1 \\
3.9 \\
2.5 \\
1.8 \\
3.0 \\
3.1 \\
4.1\end{array}$ & $\begin{array}{l}3.7 \\
3.5 \\
4.3 \\
4.0 \\
4.4 \\
2.4 \\
2.4 \\
2.7 \\
5.2 \\
4.8\end{array}$ & $\begin{array}{l}+.2 \\
+.6 \\
+1.0 \\
+1.2 \\
+1.4 \\
+1.2 \\
+1.5 \\
+1.1 \\
+.2 \\
+2.2\end{array}$ & $\begin{array}{r}1.4 \\
2.6 \\
1.5 \\
.6 \\
1.6 \\
1.2 \\
1.7 \\
1.4 \\
1.9 \\
2.3\end{array}$ & $\begin{array}{l}1.5 \\
2.0 \\
2.6 \\
3.0 \\
2.7 \\
3.5 \\
1.0 \\
2.0 \\
2.8 \\
3.1\end{array}$ & $\begin{array}{l}3.1 \\
4.5 \\
4.0 \\
3.8 \\
4.1 \\
4.9 \\
2.7 \\
3.2 \\
4.4 \\
5.7\end{array}$ & $\begin{array}{r}-.2 \\
+.1 \\
+.1 \\
-.2 \\
+.2 \\
-.2 \\
.0 \\
+.2 \\
+.3 \\
-.3\end{array}$ \\
\hline \multirow[t]{2}{*}{$\begin{array}{l}\text { Mean } \\
\text { CL }\end{array}$} & $\begin{array}{r}1.41 \\
.59\end{array}$ & $\begin{array}{r}3.39 \\
.59\end{array}$ & $\begin{array}{r}3.74 \\
.71\end{array}$ & $\begin{array}{r}+1.06 \\
.44\end{array}$ & $\begin{array}{r}1.62 \\
.40\end{array}$ & $\begin{array}{r}2.42 \\
.56\end{array}$ & $\begin{array}{r}4.04 \\
.64\end{array}$ & $\begin{array}{r}0.00 \\
.15\end{array}$ \\
\hline & $\begin{array}{r}1.2 \\
1.4 \\
4.7 \\
1.9 \\
.9 \\
2.2 \\
1.9 \\
3.7 \\
.9 \\
.7\end{array}$ & $\begin{array}{l}2.0 \\
1.3 \\
3.1 \\
3.2 \\
5.0 \\
2.8 \\
3.1 \\
3.7 \\
3.9 \\
3.0\end{array}$ & $\begin{array}{l} \\
2.7 \\
2.5 \\
2.8 \\
5.1 \\
3.7 \\
5.1 \\
4.9 \\
5.3 \\
4.7 \\
2.1\end{array}$ & $\begin{array}{l}\text { ight Env } \\
+.5 \\
+.2 \\
+5.0 \\
. .0 \\
+2.2 \\
-.1 \\
+.1 \\
+2.1 \\
+.1 \\
+1.6\end{array}$ & $\begin{array}{c}\text { ronme } \\
1.0 \\
2.3 \\
2.9 \\
2.5 \\
.9 \\
1.7 \\
2.1 \\
1.6 \\
2.7 \\
1.3\end{array}$ & $\begin{array}{l}\text { t } \\
1.9 \\
2.9 \\
1.8 \\
2.0 \\
1.1 \\
2.1 \\
2.0 \\
2.4 \\
3.5 \\
1.7\end{array}$ & $\begin{array}{l}3.0 \\
5.8 \\
4.9 \\
4.3 \\
2.1 \\
3.7 \\
4.1 \\
3.9 \\
6.1 \\
2.9\end{array}$ & $\begin{array}{r}-.1 \\
-.6 \\
-.2 \\
+.2 \\
-.1 \\
+.1 \\
.0 \\
+.1 \\
+.1 \\
+.1\end{array}$ \\
\hline $\begin{array}{l}\text { Mean } \\
\text { CL }\end{array}$ & $\begin{array}{r}1.95 \\
.93\end{array}$ & $\begin{array}{r}3.11 \\
.72\end{array}$ & $\begin{array}{r}3.89 \\
.90\end{array}$ & $\begin{array}{r}+1.17 \\
1.15\end{array}$ & $\begin{array}{r}1.90 \\
.51\end{array}$ & $\begin{array}{r}2.14 \\
.48\end{array}$ & $\begin{array}{r}4.08 \\
.91\end{array}$ & $\begin{array}{r}-.04 \\
.17\end{array}$ \\
\hline & \multicolumn{8}{|c|}{ Overall } \\
\hline $\begin{array}{l}\text { Mean } \\
\text { CL }\end{array}$ & $\begin{array}{r}1.68 \\
.51\end{array}$ & $\begin{array}{r}3.25 \\
.43 \\
\end{array}$ & $\begin{array}{r}3.82 \\
.52 \\
\end{array}$ & $\begin{array}{r}+1.11 \\
.56\end{array}$ & $\begin{array}{r}1.76 \\
.30 \\
\end{array}$ & $\begin{array}{r}2.28 \\
.34 \\
\end{array}$ & $\begin{array}{r}4.06 \\
.50 \\
\end{array}$ & $\begin{array}{r}-.02 \\
.10 \\
\end{array}$ \\
\hline
\end{tabular}

Note-PS $=$ proprioceptive shift, $V S=$ visual shift, $N A=$ negative aftereffect, $D=$ difference between $V S+P S$ and NA; positive sign indicates overadditivity, negative sign indicates underadditivity; $C L= \pm$ values for $95 \%$ confidence limits.

.05 with single targets). Also consistent with the notion of independent, but additive, components is the fact that VS and PS did not correlate significantly with each other in either the single $(-.14)$ or multiple $(.16)$ target conditions.

One other aspect of the data is worthy of note. The overadditivity with single targets appears entirely due to excessive PS. PS was greater $\left(.97^{\circ}\right)$ with a single target than with multiple targets by almost exactly the amount of overadditivity $\left(1.11^{\circ}\right)$ found with single targets. In fact, comparisons between the target conditions showed a significant difference only for the PS test, $F(1,72)=16.80, p<.001$.

These data clearly suggest that a single exposure target is one condition promoting overadditivity in hand exposure, and that the obtained overadditivity consists largely, if not entirely, in excessive PS. Consistent with the original hypothesis, it seems that, in the absence of a specific test target, subjects tend to reproduce the motor response learned during exposure training. Now, assuming that, during exposure, subjects learn to point in the direction of the prism base (i.e., opposite the displacement) in order to mark the target, and that the magnitude of this motor adjustment exceeds the true proprioceptive change, then the PS test includes a component not measured by the other tests and which appears as overadditivity. The use of multiple exposure targets minimizes such motor response learning and eliminates overadditivity due to such a factor.

The absence of any significant effects for the environment manipulation was somewhat surprising. The present data clearly suggest that the difference in test environment between experiments in the Redding and Wallace study cannot be the explanation for different magnitudes of VS. Indeed, if there was any effect at all in the present experiment, it was in the direction opposite to that predicted: i.e., greater VS with the light environment. The exact reason for this difference in VS magnitude remains elusive, but is perhaps no more mysterious than sampling error.

\section{EXPERIMENT 2}

The first experiment demonstrated that a single exposure target is one condition promoting overadditivity and identified excessive PS arising from specific motor response learning as the causal mechanism. However, this explanation cannot be applied to conditions of hall exposure, where previous research (Redding \& Wallace, 1976) found overadditivity to be more substantial than in hand exposure, but where there seems little opportunity for the acquisition of specific motor responses. It therefore seemed possible, perhaps even likely, that overadditivity has different causal bases in different experimental procedures. Experiment 2 tested the previously stated hypothesis that overadditivity may develop from an association of the single NA test target with a cognitively shifted straight-ahead such that both NA and PS are underestimated while VS is overestimated, the result being overadditivity.

\section{Method}

Subjects. As in Experiment 1, subjects were right-handed male and female volunteers from Western Illinois University, who had normal or corrected-to-normal vision and received course credit for participation; only 36 subjects were tested.

Procedure. During 15-min exposure to 20-D (base-right) prisms, subjects engaged in saggital pointing with the visible hand (hand exposure) in the same apparatus used in Experiment 1, or in hallway exploration without any view of the body (hall exposure), as in Redding and Wallace (1976). Within hand and hall exposure conditions, separate groups of nine subjects received a single straight-ahead NA test target or three simultaneously visible test targets, located straight ahead and $5^{\circ}$ to each side. Exposure fields were fully illuminated, while test fields were completely dark except for the necessary test targets. Only one exposure target was used in this experiment. 
The three tests for adaptation were the same as in Experiment 1 , except for the multiple NA targets. Order of tests was counterbalanced by a Latin square (three subjects per row in each group), and reliability was equated by having the VS and PS tests occur three times each and the subjects' pointing three times at each NA test target.

\section{Results and Discussion}

Data for individual subjects and the relevant means, together with confidence limits are shown in Table 2. An analysis of variance indicated a significant main effect for the kind of test, $F(2,64)=35.92, p<.001$, and a significant interaction of tests and number of NA test targets, $F(2,64)=5.65, p<.01$. The nature of these effects is apparent in the data averaged over exposure conditions in Table 2 . Comparisons revealed virtually perfect additivity (VS $+\mathrm{PS}=6.09^{\circ}, \mathrm{NA}=$ $5.92^{\circ}$ ) with multiple targets, $t(17)=1.28$, but significant overadditivity $\left(\mathrm{VS}+\mathrm{PS}=6.24^{\circ}, \quad \mathrm{NA}=4.40^{\circ}\right.$, difference $=1.84^{\circ}$ ) with a single test target, $t(17)=5.23$, $\mathrm{p}<.001$. As in Experiment 1, this difference between test target conditions is particularly compelling since it is reflected in the data for individual subjects. With a single target, 14 of the 18 subjects showed overadditivity, while only half the subjects showed overadditivity with multiple targets. Moreover, deviation from additivity for individual subjects with multiple targets was quite small, indicating random variation around zero, while the overadditivity for single target conditions was substantial for most subjects.

The only other source of variance in these data which was significant was the interaction of kind of test and exposure condition, $F(2,64)=20.06, p<.001$. Thus, as has been found in previous studies (Redding, 1978; Redding \& Wallace, 1976), VS is greatest with hall exposure and PS is greatest with hand exposure, but this effect does not interact with the number of test targets, $F(2,64)=0.02$, and consequently does not qualify the effect of this latter variable on additivity.

The conclusion of greater additivity with multiple test targets was also supported by rank-order correlational analysis, as was the case in the first experiment, although the level of correlation was generally less. The NA test correlated more highly with VS $(.59$, $p<.02)$ and PS $(.55, p<.02)$ in multiple-target conditions than in single-target conditions $(.42, \mathrm{p}<.10$, and $.49, \mathrm{p}<.05$, respectively), and, consistent with the notion of independent components, VS and PS did not correlate significantly with each other in either the single (-.15) or multiple (-.14) target conditions.

These data are consistent with our initial hypothesis, and suggest that overadditivity in both hand and hall exposure arose from the NA being artificially reduced by the presence of a cognitive shift in egocentric straight-ahead and association of the single NA test target with this cognitively shifted direction. In this connection, it is interesting to note that the only significant comparison between target conditions for the
Table 2

Level of Adaptation in Degrees for Subjects Receiving Hall or Hand Exposure During 20-D (Base-Right) Displacement Under Conditions of Single or Multiple Test Targets



Note $-P S=$ proprioceptive shift, $V S=$ visual shift, $N A=$ negative aftereffect, $D=$ difference between $V S+P S$ and $N A$; positive sign indicates overadditivity, negative sign indicates underadditivity; $C L= \pm$ values for $95 \%$ confidence limits.

three kinds of tests was for the NA test, $F(1,64)=$ $14.25, \mathrm{p}<.001$. The NA was reduced from $5.92^{\circ}$ for multiple targets to $4.40^{\circ}$ for the single target, while the other tests were essentially unaffected by the change in number of test targets. Thus, it seems reasonable to assume that overadditivity was eliminated by removing the association between the NA target and the cognitive straight-ahead, not by eliminating the straightahead shift per se. In the latter case, if use of multiple NA targets eliminated straight-ahead shift, VS would decrease and PS would increase when the straight-ahead shift influence was removed.

\section{CONCLUSIONS}

These experiments have established the reliability of the overadditivity effect, and have identified singleexposure targets and/or single NA test targets as conditions promoting its occurrence. Moreover, evidence is provided for two causal bases for overadditivity; specific motor response learning with single-exposure targets 
and the association of a single NA test target with a cognitive shift in egocentric straight-ahead. ${ }^{2}$ Thus, overadditivity seems to have multiple causes, but the use of multiple (exposure and/or test) targets appears to be a general remedy for the problem, and there is no reason to assume that adaptive components combine in other than simple linear fashion.

\section{REFERENCES}

HARRIS, C. S. Adaptation to displaced vision: Visual, motor, or proprioceptive change? Science, 1963, 140, 812-813.

HaRris, C. S. Beware the straight-ahead shift: A nonperceptual change in experiments on adaptation to displaced vision. Perception, 1974, 3, 461-474.

HAY, J. C., \& PiCK, H. L. Visual and proprioceptive adaptation to optical displacement of the visual stimulus. Journal of Experimental Psychology, 1966, 72, 419-444.

Mclaughlin, S. C., \& Webster, R. G. Changes in straightahead eye position during adaptation to wedge prisms. Perception \& Psychophysics, 1967, 2, 37-44.

Redding, G. M. Additivity in adaptation to optical tilt. Journal of Experimental Psychology: Human Perception \& Performance, 1978, 4, 178-190.

Redding, G. M., \& Wallace, B. Components of displacement adaptation in acquisition and decay as a function of hand and hall exposure. Perception \& Psychophysics, 1976, 20, 453-459.

Templeton, W. B., Howard, I. P., \& Wilkinson, D. A.
Additivity of components of prismatic adaptation. Perception \& Psychophysics, 1974, 15, 249-257.

WAllace, B. Stability of Wilkinson's linear model of prism adaptation over time for various targets. Perception. 1977, 6, 145-151.

Wallace, B., \& GarretT, J. B. Perceptual adaptation with selective reduction of felt sensation. Perception, 1975, 4. 437-445.

Welch, R. B., Choe, C. S., \& Heinrich, D. R. Evidence for a three-component model of prism adaptation. Journal of Experimental Psychology, 1974, 103, 700-705.

Wilkinson, D. A. Visual-motor control loop: A linear system? Journal of Experimental Psychology, 1971, 89, 250-257.

\section{NOTES}

1. Per convention, positive signs indicated change in the adaptive direction and negative signs were used to indicate maladaptive shifts. The adaptive direction is toward the prism base for NA and PS tests, and in the direction of displacement for the VS test.

2. It may be noted that the present experiments provide no direct evidence of either response or cognitive shift. We are not aware that such direct evidence is available. However, to the extent that the present data and other observations (Harris, $1963,1974)$ are consistent with prediction based on such assumptions, there is relatively strong indirect evidence for the presence of such phenomena in adaptation studies.

(Received for publication December 20, 1977; revision accepted April 14, 1978.) 